Umweltschuiz im globalen Wettbewerb

\title{
Neue Spielregeln für den Unternehmenserfolg
}

\begin{abstract}
Die politischen und gesellschaftlichen Steverungsmuster befinden sich im Zuge der Globalisierung in einem Umbruch. Für die Chancen und Grenzen von Unternehmen, Umweltschutz im globalen Wettbewerb umzusetzen, hat dies weitreichende Folgen. Immer mehr ist die "grenzenlose Unternehmung" gefragt, die nicht nur bezogen auf ihre Standortwahl, sondern auch hinsichtlich ihrer wirtschafts- und umweltpolitischen Rolle Grenzen überschreitet.
\end{abstract}

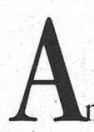

Von Klaus Fichter und

Uwe Schneidewind internationalen Verflechtungen mehr und mehr zu einer bestimmenden Herausforderung für die umwelt- und wirtschaftspolitischen Akteure. Vorangetrieben durch die Entstehung globaler Informationssysteme und Kommunikationsnetze ist die Globalisierung der Wirtschaft einer der bedeutendsten Aspekte der derzeitigen Neuordnung der Welt. Diese Entwicklung ist einerseits durch den zunehmenden Einfluß multinationaler Unternehmen und der Finanzmärkte und andererseits durch den abnehmenden Handlungsspielraum der nationalen Umwelt- und Wirtschaftspolitik geprägt.

In der aktuellen Diskussion wird immer wieder auf die ,Grenzen des Wettbewerbs“ (1) hingewiesen und vor einer. Wettbewerbsgläubigkeit gewarnt, die übersieht, daß freie Märkte die weltweit wachsenden sozialen, ökologischen, demographischen und beschäftigungspolitischen Probleme nicht lösen können, sondern diese Effekte häufig gerade die nicht beabsichtigten Nebenfolgen des ökonomisch getriebenen Globalisierungsprozesses sind (2). ,Wer ausschließlich auf den Markt setzt, zerstört mit der Demokratie auch die Marktwirtschaft selbst."

(3) Folgt man der Argumentation dieser Globalisierungskritik, wie sie von den genannten Autoren anschaulich und anhand von umfassenden empirischen Material sowie konzeptionellen Überlegungen vorgebracht wird, scheint auf globaler Ebene das Primat der Politik zunehmend zurïckgedrängt zu werden. Die demokratische politische Steuerbarkeit wirtschaftlicher Prozesse ist in Frage gestellt.

Die gesellschaftliche Debatte über diese Entwicklungen ist in vollem Umfang entbrannt. For- derungen nach neuen Regulierungen der globalen Finanzmärkte, die Integration von sozialen und ökologischen Kriterien in das Regelwerk des Welthandelsabkommens, die Stärkung supranationaler Institutionen wie der UN stehen auf der Agenda der aktuellen politischen Diskussion. Es geht bei diesen Bemühungen darum: 1. neue globale Ordnungsstrukturen und Spielregeln zu etablieren, die die negativen externen Effekte eines intensivierten Wettbewerbsprozesses verringern sowie

2. Umweltschutzanforderungen fest in das Marktgeschehen und den Wettbewerbsprozeß zu integrieren.

Bei der Suche nach diesen neuen ,Global governance“- Strukturen wird deutlich, daß diese sich nicht nach den Mustern klassischer Politikformen ausgestalten lassen, d.h. in Form demokratisch legitimierter Zentralgewalten, die Rahmenordnungen festlegen, in denen sich das Handeln der Akteure in Wirtschaft und Gesellschaft abspielt. Die Schwierigkeiten eines entsprechenden Vorgehens zeigen sich schon auf der Ebene der Europäischen Union, im globalen Maßstab scheinen sie auf absehbare Zeit ohnehin nicht realisierbar. An die Stelle der Hierarchie treten in Zukunft wechselseitige Abhängigkeiten in einer Heterarchie verschiedener Akteure, die eine Koordination in der Horizontalen zwischen Partnern mit eigener Verantwortung, Interessen und Kompetenzen erfordert (4). Neben Umweltschutzorganisationen oder Normungsvereinigungen werden Unternehmen und Unternehmensverbände selbst einen wichtigen Anteil an der Ausgestaltung der neuen Steuerungsstrukturen haben.

Für Unternehmen hat dies weitreichende Folgen. Die Veränderung der politischen und gesellschaftlichen Regulierungsmuster fuihrt dazu, daß neue Kräfte im Wettbewerb relevant werden. Hierzu gehören z.B. der Einfluß großer internationaler Umweltschutzorganisationen, institutioneller Anleger und Bewertungsagenturen auf den Finanzmärkten oder der Massenmedien. Diese neuen Kräfte gestalten Märkte um, schaffen neue Märkte und stellen erhebliche Anforderungen an die Wettbewerbsstrategien von Unternehmen. Im ökologischen Kontext kommt zwei Strategieformen dabei eine Schlüsselbedeutung zu: geeigneten Formen der Umweltkommunikation sowie neuen Kooperationsmustern insbesondere entlang von Wertschöpfungsketten. Die theoretische Reflexion dieser Umbruchsprozesse steht erst am Anfang. Um Unternehmen notwendige Orientierungspunkte zu geben, kommt hier auf eine sozialwissenschaftlich aufgeklärte Managementlehre ein erheblicher Forschungsbedarf zu. Abbildung 1 gibt die gerade skizzierte Umbruchslogik im Überblick wieder. Die sechs einzelnen Elemente, die alle Themen der IÖW/VÖW-Konferenz sein werden, (vgl. Kasten S. 11) werden im folgenden näher beleuchtet.

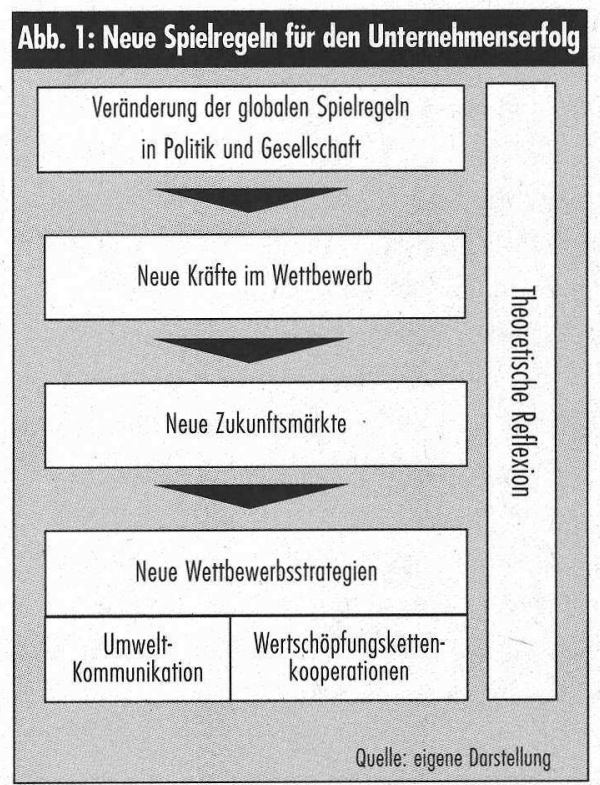

\section{Globale Spielregeln}

Die abnehmende Steuerungsfähigkeit der Nationalstaaten und damit das Fehlen zentraler Steuerungsinstanzen wird spätestens mit dem Bericht der Brandt-Kommission debattiert und in diesem Rahmen das Konzept der Global Governance entwickelt (5). Hinter diesem Konzept steht die Vorstellung, daß es mehr und mehr auf die Steuerungsfähigkeit unterschiedlicher Akteure auf der politischen Weltbühne ankommt. Dabei spielen auch weiterhin Nationalstaaten eine wesentliche 


\title{
Umweltschutz im globalen Wettbewerb - Neue Spielregeln für den Unternehmenserfolg
}

\author{
Schirmberrschaft: Bundesumweltministerium. In Zusammenarbeit mit \\ dem Umweltprogramm der Vereinten Nationen (UNEP), B.A.U.M., \\ future e.V. und mit freundlicher Unterstützung der \\ BSH Bosch und Siemens Hausgeräte GmbH
}

\section{Schwerpunkte:}

Zukunftsmärkte und neue Wettbewerbsstrategien

- Wie lassen sich Markterfolg und Umweltschutz in Wettbewerbsstrategien gezielt und daverhaft verbinden? Bsp.: Der Ausstieg von BSH Bosch und Siemens Housgeröte ous der FCKW- und FKW-Technologie als Erfolgsfaktor in globalen Märkten

- Welche Umweltkooperationen dienen der Entwicklung von Zukunftsmärkten?

Bsp:: Die Zusammenarbeit von WWF und Unilever bei der Entwicklung eines Nachhaltigkeitssiegels für die Fischwirtschaft

Erfolgsfaktor Umweltkommunikation

- Wie können Umweltthemen in der Marktkommunikation und Öffentlichkeitsarbeit effolgreich verankert werden?

Bsp.: Die umweltorientierte Verbraucherkommunikation als Element der Wettbewerbsstrategie bei Tengelmann

- Wie verändern luK-Technologien Umwellschutzmärkte?

Bsp.: Altautorecyclingbörsen im Internet

Gemeinsam wettbewerbsfähig - Wie lassen sich durch Kooperationen in der Wertschöpfungskette umweltschonende und markffähige Produkte entwickeln?

Bsp.: Öko-Kaffee im Großkundengeschäft bei Kraft, Jacobs, Suchard

Bsp.: Von der Zeitung bis in den Wald: Ökologische Optimierung der Papierkette

Neve Kräfte im globalen Wettbewerb - Welchen Einfluß haben die Medien, NGO's und Finanzdienstleister ouf Wettbewerbsprozesse und Markterfolg?
Bsp.: Startschuỉ für den "Sustainablity-Dow-Jones" - ein never Index am Weltfinanzmarkt

Bsp.: Öko-Rankings in Massenmedien - Die Umweltcomputerliste in Computer-Bild

Globale Spielregeln für Umwelischutz im Wettbewerb In welche Richtung entwickeln sich die Welthandelsordnung oder Vereinbarungen über ausländische Direktinvestitionen? Welche Rolle spielen multinationaler Unternehmen in der Global Governance?

Die Grenzenlose Unternehmung: Empirie und Theorie Welche Folgen haben luK-Techniken und die Virtualisierung der Wirtschaft für den Umweltschutz? Wie setzen weltweit agierende Unternehmen ihre Umweltschutzstrategien um?

In über 30 Einzelveranstaltungen werden zahlreiche prominenten Referenten aus Wirtschaft, Politik und Wissenschaft vortragen

\section{Akłueller Programmstand und Information im Internet:}

\section{www.ioew.de/konferenz}

Konferenzleitung: Dr. Klaus Fichter, IÖW und Prof. Dr. Uwe Schneidewind, Vöw

Kontakt: IÖW, Christiane Ballschuh, Tel. 030/ 8845 94-16, Fax 030/8 825439 , E-mail: Christiane.Ballschuh@ioew.de

schieden wird: Neue Kräfte treten auf den Märkten auf. Hierzu gehören der Einfluß von Medien, die Arbeit von Umweltschutz- und anderen Nichtregierungsorganisationen sowie von Dienstleistern in den Finanzmärkten. Die verhinderte Versenkung der Ölplattform Brent Spar oder die durch internationale NGO-Zusammenarbeit erreichte Blockade des multilateralen Investitionsabkommens MAI vermitteln Eindrücke des hier neu entstehenden Einflusses. Ermöglicht wird die Macht der internationalen NGO's insbesondere durch die geschickte Nutzung massenmedialer Kommunikationsmuster. Die Massenmedien werden in der globalisierten Welt zum entscheidenden Transmissionsriemen von Wettbewerbsveränderungen (vgl. den Beitrag von Zerfaß in diesem Heft).
Neben den Massenmedien wirken die Finanz- und Kapitalmärkte, als die in der Globalisierung am weitesten fortgeschrittenen Institutionen, am stärksten auf das Handeln von Unternehmen im globalen Wettbewerb zuriick. In dem Maße, in dem die Akteure (wie Banken, z.B. institutionelle Anleger oder Rating-Agenturen) in diesen Märkten ökologische Aspekte in ihr Handeln einbeziehen, verändern sich ebenfalls die Durch- und Umsetzungschancen ökologischer Strategien im globalen Wettbewerb. Gerade in den letzten Jahren zeigen sich in diesem Bereich rasante Entwicklungen (vgl. u.a. den Beitrag von Flatz).

\section{Zukunftsmärkte und neve Wettbewerbsstrategien}

Während der Zusammenhang von Ökologie und Wettbewerbsfähigkeit bis Anfang der 90er Jahre zumeist als Kostenproblem begriffen wurde, werden seit einigen Jahren die Profilierungschancen und Kostensenkungspotentiale des Umweltschutzes betont. Beide Sichtweisen sind nicht falsch, erweisen sich aber bei näherer Betrachtung als einseitig und verkürzt, denn sie gelten jeweils nur für bestimmte Situationen. Der Zusammenhang ist weder durch ein einfaches Harmoniemodell (Umweltschutz macht sich bezahlt) noch durch ein reines Konfliktmodell (Gewinn oder Umweltschutz) adäquat zu erfassen. Erfolgreiche Handlungsstrategien verlangen differenziertere Betrachtungsweisen (vgl. dazu den Beitrag von Dyllick).

Besonders wichtig ist es in diesem Zusammenhang zu erkennen, welche ganz neuen Märkte durch neue Steuerungsmuster in der Umwelffrage entstehen und wie die bestehenden Wettbewerbsstrategien an die veränderten Einflußkonstellationen anzupassen sind (vgl. dazu den Beitrag von Bärmann). Kommunikation und Kooperation werden dabei zu bedeutenden Schlïsselkompetenzen.

\section{Umweltkommunikation als Erfolgsfaktor}

Durch die zunehmende Arbeitsteiligkeit der Weltwirtschaft, die rasante Entwicklung bei Informations- und Kommunikations (IuK)-Technologien und die Herausbildung einer globalen Mediengesellschaft ist die Bedeutung von Information und Kommunikation für die Wettbewerbsfähigkeit von Unternehmen in den vergangenen Jahren permanent gestiegen. Sie spielen heute bei Marktdynamik, Wettbewerb sowie in politischen und öffentlichen Meinungsbildungsund Entscheidungsprozessen eine entscheiden-

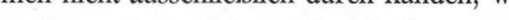
werber und staatliche Rahmenbedingungen ent- 
de Rolle. Durch IuK-Technologien verändern sich Unternehmen und Märkte in rasantem Tempo (z.B. virtuelle Unternehmen, Tele-Shopping, Teledienstleistungen usw.; vgl. dazu den Beitrag von Picot). Durch die Komplexität und „Unübersichtlichkeit“ wirtschaftlicher und gesellschaftlicher Vorgänge erlangt außerdem die Unternehmenskommunikation eine zentrale operative wie strategische Bedeutung. Eine systematische und auf die Unternehmensstrategie abgestimmte Verknüpfung von Marktkommunikation, interner Kommunikation und Öffentlichkeitsarbeit wird zunehmend bedeutsamer für die Wettbewerbsfähigkeit.

\section{Gemeinsam wettbewerbsfähig durch Kooperation}

Umweltschutzstrategien erstrecken sich heute in der Regel auf die gesamte Wertschöpfungskette. Kunden und Umweltschutzorganisationen nehmen Unternehmen für den gesamten Lebensweg ihres Produktes in die Pflicht. Recyclinganforderungen oder die Substitution von bedenklichen Einsatzstoffen lassen sich nur durch enge Kooperationen von Partnern entlang der Wertschöpfungskette einlösen. Umweltschutz und Wettbewerbsfähigkeit verbinden daher die Unternehmen am besten, die es verstehen solche Formen der Zusammenarbeit effektiv und effizient zu organisieren. Erst seit wenigen Jahren ensteht das entsprechende Wissen über ein solches „Stoffstrommanagement" (6) und verbreitet sich über einzelne Branchen hinaus.

\section{Theoretische Reflexion}

Trotz der zunehmenden Globalisierung von Unternehmen und Branchen wurde dieses Phänomen bisher mit seinen Konsequenzen auf das Umweltengagement von Unternehmen und die damit verbundenen Wettbewerbswirkungen kaum aus betriebswirtschaftlicher Perspektive analysiert. Die Forschung steht hier am Anfang. Besonders fruchtbar scheint es dabei, Theorieentwicklungen aus der aktuellen Managementlehre aufzugreifen und auf die Fragestellung von „Umweltschutz im globalen Wettbewerb“ anzuwenden. Dazu müssen die derzeitig vorherrschenden „Schulen“ der unternehmensbezogenen Wettbewerbstheorie (der marktorientierte und der ressourcenorientierte Ansatz; zu letzterem vgl. den Beitrag von Fischer/ Pfriem) einer Prüfung unterzogen werden (7). Nur wenn diese Integration gelingt, wird die Managementleh-

\section{Thesen zu Umweltschutz im globalen Wetthewerb}

1. Ob in den kommenden Jahrzehnten im globalen Maßsstab Schritte in Richtung einer nachhaltigen Entwicklung gelingen, wird in starken Maße davon abhängen, ob die Umwellschutzleistung zu einem wesentichen Faktor für den Wettbewerbserfolg von Unternehmen wird. Nur cuf diese Weise werden Unternehmen ihre ganze Kreativ- und Produkivkruft in den Dienst eines sozial-ökologischen Strukturwandels stellen können. Der Rahmen für einen funktionierenden Wettbewerb der Umweltschutzleistung von Unternehmen ist derzeit nur sehr eingeschränkt gegeben. Nlassische Regulierungsmuster treten bei der Rahmensetzung in Zukunft weiter in den Hintergrund. Die Finanzmärkte und neue informatorische Regelungen und Lösungen werden dagegen eine zentrale Rolle bei der Schaffung eines geeigneten Rahmens spielen.

2. Um die Umweltschutzleistung gesamter Unternehmen in marktlichen Prozessen zu stärken, bedarf es der Generierung neuer Informationstatbestände und never Informationsflüsse (Umweltberichterstattung, Stoffstrommanagement etc.). Auf diese Weise können Ineffizienzen in der Werischöpfungskette aufgedeckt und innovalive Lösungen gefördert werden. Umweltschutzkriterien und neve Informationen verändern den Selektionsmechanismus am Markt und schaffen für NGO's und Medien neue Möglichkeiten in Wettbewerbsprozesse einzugreifen.

3. Angesichts der vielfältigen Handlungs- und Gestaltungsspielrüume, die Unternehmen sowohl in ihren internen wie externen Interaktionen nutzen können, ist das herkömmliche

re dem Anspruch gerecht werden, anschlußfähiges Wissen für Unternehmen zu generieren, daß diesen hilft, die „,neuen Spielregeln für den Unternehmenserfolg" zu verstehen. Die Thesen (vgl. Kasten) bieten hierzu einen ersten Anhaltspunkt.

\section{Anmerkungen}

(1) Die Gruppe von Lissabon: Grenzen des Wettbewerbs, Die Globalisierung der Wirtschaft und die Zukunft der Menschheit. München 1997

(2) Vgl. z.B. Altvater, E./ B. Mahnkopf: Grenzen der Globalisierung. Münster 1996, und

Martin, H.P.; H. Schumann: Die Globalisierungsfalle. Der Angriff auf Demokratie und Wohlstand. Reinbek 1996.

(3) Beck, U.: Was ist Globalisierung? Frankfurt a.M.

1997, S. 196.

(4) Vgl. Steger, U. (Hrsg.): Wirkmuster der Globalisierung: Nichts geht mehr, aber alles geht. Bericht des Ladenburger Kollegs "Globalisierung verstehen und gestalten". Ladenburg 1998, S. 29ff.

(5) Stiftung Entwicklung und Frieden ( $\mathrm{Hg}$. ): Nachbarn in Einer Welt. Bonn 1995. Vgl. auch den Schwerpunkt 2/98 von Ökologisches Wirtschaften. ökonomische Verhaltensmodell, in dem Unternehmen als extern gesteverte Reiz-Reaktions-Maschinen begriffen werden, zu revidieren. Für die gezielte Gestaltung des Verhültnis von Ökologie und Wettbewerbstähigkeit ist dem Wechselspie von Handeln und Strukturen besondere Aufmerksamkeit zu schenken. Unternehmen haben Einfluß auf die Bedingungen und Regeln des Wetthewerbs. Sie sind daher als "strukturpolitische Akteure" (8) gefordert.

4. Es ist die "grenzenlose Unternehmung"(9), um die sich die Frage nach Umwelischutz im globalen Wettbewerb dreht:

- Die globalen, durch die luK-Möglichkeiten zunehmend standort- und unternehmensstruktur-unabhängig agierenden (multinationalen) Konzerne sind ein zentraler Grund für die politischen Steverungsdefizite, die sich im Zuge der Globalisierung stellen.

- Gleichzeitig eröffnet die so verstandene Grenzenlosigkeit ganz neue lösungspotentiale. Das Schmieden von ökologisch orientierten Kooperationen zwischen Unternehmen, aber ouch mit Anspruchsgruppen wird im virtuellen Roum in kürzester Zeit möglich und eröffnet neve Gestaltungsmöglichkeiten.

- "Grenzenlos" müssen Unternehmen aber auch im Hinblick ouf ihr Rollenverständnis sein. Es beschränkt sich in den "governance"-Strukturen der Zukunft immer weniger nur auf eine Marktrolle. In der globalen "Netz werkgesellschaft" (10) sind Unternehmen zunehmend auch politische und gesellschaftliche Akteure und bekennen sich proaktiv zu dieser Herousforderung.

(6) Vgl. Friege, H. u.a.: Das Management von Stoffströmen. Berlin U.a. 1998.

(7) Vgl. Fichter, K.: Umweltkommunikation und Wettbewerbstähigkeit. Marburg 1998, S. $114 f f$.

(8) Schneidewind, U.: Die Unternehmung als strukturpolitischer Akteur. Marburg 1998.

(9) Picot, A./ R. Reichwald/ R. Wigand: Die grenzenlose Unternehmung. 2. Aufl., Wiesbaden 1996.

(10) Messner, D.: Die Netzwerkgesellschaft. Köln 1995.

\section{Die Autoren}

Dr. Klaus Fichter ist Leiter der Forschungsgruppe "Ökologische Unternehmenspolitik“ beim Institut für ökologische Wirtschaftsforschung (IÖW), Berlin. Prof. Dr. Uwe Schneidewind ist Professor für Produktionswirtschaft und Umwelt an der Universität Oldenburg und Vorsitzender der Vereinigung für ökologische Wirtschaftsforschung (VÖW e.V.).

Kontakt: IÖW, Giesebrechtstr. 13, 10629 Berlin. Tel. 030/88 4594-0, E-mail: Klaus.Fichter@ioew.de 
(c) 20I0 Authors; licensee IÖW and oekom verlag. This is an article distributed under the terms of the Creative Commons Attribution Non-Commercial No Derivates License (http://creativecommons.org/licenses/by-nc-nd/3.o/), which permits unrestricted use, distribution, and reproduction in any medium, provided the original work is properly cited. 